\title{
The Variational Principle for the Uniform Acceleration and Quasi-Spin in Two Dimensional Space-Time ${ }^{\star}$
}

Roman Ya. MATSYUK

Institute for Applied Problems in Mechanics and Mathematics, 15 Dudayev Str., L'viv, Ukraine E-mail: matsyuk@lms.lviv.ua

Received October 31, 2007, in final form January 18, 2008; Published online February 06, 2008

Original article is available at http://www.emis.de/journals/SIGMA/2008/016/

\begin{abstract}
The variational principle and the corresponding differential equation for geodesic circles in two dimensional (pseudo)-Riemannian space are being discovered. The relationship with the physical notion of uniformly accelerated relativistic particle is emphasized. The known form of spin-curvature interaction emerges due to the presence of second order derivatives in the expression for the Lagrange function. The variational equation itself reduces to the unique invariant variational equation of constant Frenet curvature in two dimensional (pseudo)-Euclidean geometry.
\end{abstract}

Key words: covariant Ostrohrads'kyj mechanics; spin; concircular geometry; uniform acceleration

2000 Mathematics Subject Classification: 53A40; 70H50; 49N45; 83C10

\section{Introduction}

It turns out that the notion of the uniformly accelerated relativistic test particle world line [1] coincides with the notion of the geodesic circle in pseudo-Riemannian geometry [2]. In two dimensions both these notions may be equivalently replaced by the only condition that the first (and the only) Frenet curvature $k$ of the curve in the consideration keeps constant along this curve (in physical terms, along the world line of the test particle).

In the natural parametrization by the arc length $s$ (in physical terms, by proper time) all the notions, mentioned above, amount to the following third order differential equation (cf. appendix A.5)

$$
\frac{D^{3} x^{l}}{d s^{3}}+g_{m n} \frac{D^{2} x^{m}}{d s^{2}} \frac{D^{2} x^{n}}{d s^{2}} \frac{D x^{l}}{d s}=0 .
$$

The left hand side of this equation is known in the physical literature under the name of the Abraham vector. This vector is believed to adequately represent the notion of the relativistic acceleration of test particle [1]. Of course, the notion of geodesic circles does not depend on any arbitrary reparametrization $s(\xi)$ of the independent variable along the curve in (1.1).

One may ask whether geodesic circles could be the extremals of some variational problem. Since the equation (1.1) involves third order derivatives, it is appropriate to speak of higher order variational calculus. Rather trying in naïve way to find a Lagrange function for the equation (1.1) (or, better, an equivalent equation), it may happen more efficient to set a general problem of finding all possible third order variational equations in two-dimensional space which keep the

${ }^{\star}$ This paper is a contribution to the Proceedings of the Seventh International Conference "Symmetry in Nonlinear Mathematical Physics" (June 24-30, 2007, Kyiv, Ukraine). The full collection is available at http://www.emis.de/journals/SIGMA/symmetry2007.html 
Frenet curvature $k$ constant along their solutions. Of course, one may hope to solve in reasonable fullness such an inverse problem only if restricted to certain types of the space geometry. The natural restriction with regard to (pseudo)-Riemannian space should, of course, be that of its local model - the (pseudo)-Euclidean space. Thus one comes to the necessity of the formulation of the invariant inverse variational problem possessing the first integral $k$. This problem in two dimensions received the ultimate answer in [3] which is quoted below as Proposition 2. This proposition says that to obtain geodesic circles, one should, in two dimensions, necessarily handle the Frenet curvature itself as the integrand of the variational functional.

The fashion of including Frenet curvatures into the variational integrand in physics literature, initiated, in our opinion, by the papers of Plyushchay and Nesterenko, counts 30 years by now. Still, sometimes it escapes the common knowledge that such quantities as, say, Frenet curvatures, are always constants of motion along the extremals of the variational problems where the Lagrange functions depend on nothing but the corresponding curvatures themselves [4]. This fact being noticed long ago [5] for the (pseudo)-Euclidean case, we present it here in general framework by means of rather trivial observation of Proposition 1. Unfortunately, only in two dimensions the first Frenet curvature taken as the Lagrange function produces, due to its linearity in second derivatives, a third order equation.

In Section 3.2 we deduce the variational equation from the variational functional

$$
\int k d \xi+d s
$$

in (pseudo)-Riemannian two-dimensional space and prove that the set of its extremals includes all geodesic circles.

Also we discovered quite an interesting fact, in our opinion, that the force on the particle, having the same form as that produced by spin-curvature interaction, emerges as a simple result of calculating the variation of second derivatives in the variational integrand.

\section{Preliminaries}

\subsection{Parameter independence and Ostrohrads'kyj mechanics}

The right action of the prolonged group $G L_{(2)}(\mathbb{R}) \stackrel{\text { def }}{=} \tilde{J}_{0}^{2}(\mathbb{R}, \mathbb{R})_{0}$ of parameter transformations (invertible transformations of the independent variable $\xi$ ) on the second order velocities space $T^{2} M=\left\{x^{n}, u^{n}, \dot{u}^{n}\right\}$ gives rise to the so-called fundamental fields on $T^{2} M$ (cf. Appendix A.1)

$$
\zeta_{1}=u^{n} \frac{\partial}{\partial u^{n}}+2 \dot{u}^{n} \frac{\partial}{\partial \dot{u}^{n}}, \quad \zeta_{2}=u^{n} \frac{\partial}{\partial \dot{u}^{n}} .
$$

A function $f$ defined on $T^{2} M$ does not depend on the change of independent variable $\xi$ (so-called parameter-independence) if and only if

$$
\zeta_{1} f=0, \quad \zeta_{2} f=0 .
$$

On the other hand, a function $L$ defined on $T^{2} M$ constitutes a parameter-independent variational problem with the functional $\int L\left(x^{n}, u^{n}, \dot{u}^{n}\right) d \xi$ if and only if the following Zermelo conditions are satisfied [6, formula (8.19)]

$$
\zeta_{1} L=L, \quad \zeta_{2} L=0 .
$$

Note 1. At this point it worths mentioning the difference between the conditions (2.2) and (2.3). It would be instructive not to confuse the idea of the parameter independence of a variational 
problem with that of the parameter independence of Lagrange function. The Lagrange function of a parameter-independent variational problem in not parameter-independent itself.

The formulation of Zermelo conditions in the most general case of arbitrary order and of arbitrary number of independent variables (Zermelo-Géhéniau) may be read from [7] whereas the corresponding proof may be found in [8] as well as in [5].

Let us introduce the generalized momenta

$$
p_{n}^{(1)}=\frac{\partial L}{\partial \dot{u}^{n}}, \quad p_{n}=\frac{\partial L}{\partial u^{n}}-\frac{d p_{n}^{(1)}}{d \xi} .
$$

The Euler-Poisson equation is given by

$$
\mathcal{E}_{n}=\frac{\partial L}{\partial x^{n}}-\frac{d p_{n}}{d \xi}=0
$$

The Hamilton function is given by

$$
H=p_{n}^{(1)} \dot{u}^{n}+p_{n} u^{n}-L .
$$

Lemma $1([5,9])$.

$$
H=\zeta_{1} L-\frac{d \zeta_{2} L}{d \xi}-L
$$

Proposition 1. If a function $L_{\mathrm{II}}$ is parameter-independent and if a function $L_{\mathrm{I}}$ constitutes a parameter-independent variational problem, then $L_{\mathrm{II}}$ is constant along the extremals of $L=L_{\mathrm{II}}+L_{\mathrm{I}}$.

Proof. By Lemma 1 and in force of the properties (2.2) and (2.3) we calculate $H_{L_{\mathrm{II}}+L_{\mathrm{I}}}=$

$\zeta_{1}\left(L_{\mathrm{II}}+L_{\mathrm{I}}\right)-\frac{d}{d \xi} \zeta_{2}\left(L_{\mathrm{II}}+L_{\mathrm{I}}\right)-L=-L_{\mathrm{II}}$. But as far as the Hamilton function is constant of motion, so is the $L_{\mathrm{II}}$.

\subsection{Inverse variational problem for geodesic circles}

As stated in the previous papers by the author, the general third-order variational equation takes on the shape $[10,11]$

$$
A_{m n} \ddot{u}^{n}+\dot{u}^{l} \partial_{u^{l}} A_{m n} \dot{u}^{n}+B_{m n} \dot{u}^{n}+c_{m}=0,
$$

where the skew-symmetric matrix $A$, the matrix $B$, and a column $c$ all depend on $\xi, x^{n}$, and $u^{n}$, and satisfy the following system of partial differential equations

$$
\begin{aligned}
& \partial_{u}[m \\
& 2 B_{n k]}=0, \\
& 2 \partial_{u}[m]-3 B_{n] k}-4 \partial_{x} A_{m n}=0, \\
& \partial_{u} A_{n] k}+\partial_{x^{k}} A_{n)}-\mathbf{D}_{\mathbf{1}} B_{(m n)}=0,2 \mathbf{D}_{\mathbf{1}} \partial_{u^{k}} A_{m n}=0, \\
& 2 \partial_{u^{k}} \partial_{u}\left[m c_{n]}-4 \partial_{x}\left[m B_{n] k}+\mathbf{D}_{\mathbf{1}}{ }^{2} \partial_{u^{k}} A_{m n}+6 \mathbf{D}_{\mathbf{1}} \partial_{x}\left[m A_{n k]}=0,\right.\right.\right. \\
& 4 \partial_{x}\left[m c_{n]}-2 \mathbf{D}_{\mathbf{1}} \partial_{u}\left[m c_{n]}-\mathbf{D}_{\mathbf{1}}{ }^{3} A_{m n}=0 .\right.\right.
\end{aligned}
$$

Here the differential operator $\mathbf{D}_{\mathbf{1}}$ is the lowest order truncated operator of total derivative, $\mathbf{D}_{1}=\partial_{\xi}+u^{l} \partial_{x^{l}}$. We refer to the appendix A.2 on some additional comments concerning the nature of the variationality conditions (2.7). 
On the other hand, we are interested only in differential equations which enjoy the (pseudo)Euclidean symmetry. Because of that in two dimensions the skew-symmetric matrix $A$ in (2.6) is invertible, there is no problem to formulate the concept of the symmetry in the way

$$
\left.\left(X \mathcal{E}_{n}\right)\right|_{\mathcal{E}=0}=0
$$

where $\mathcal{E}$ denotes the left hand side of the equation (2.6) and $X$ stands for the infinitesimal generator of the (pseudo)-Euclidean group.

The system of partial differential equations (2.7) together with (2.8) was solved in [3] and the following result was established:

Proposition 2. Let some system of third order differential equations (2.6) enjoy the following properties:

- the conditions (2.7) are satisfied;

- the system (2.6) possesses Euclidean symmetry according to (2.8);

- the Euclidean geodesics $\dot{\boldsymbol{u}}=\mathbf{0}$ enter in the set of solutions of (2.6);

- $\frac{d k}{d \xi}=0$ along the solutions of $(2.6)$.

Then

$$
\mathcal{E}_{n}=\frac{\epsilon_{n l} \ddot{u}^{l}}{\|\boldsymbol{u}\|^{3}}-3 \frac{(\dot{\boldsymbol{u}} \cdot \boldsymbol{u})}{\|\boldsymbol{u}\|^{5}} \epsilon_{n l} \dot{u}^{l}+m \frac{(\boldsymbol{u} \cdot \boldsymbol{u}) \dot{u}_{n}-(\dot{\boldsymbol{u}} \cdot \boldsymbol{u}) u_{n}}{\|\boldsymbol{u}\|^{3}} .
$$

In the above statement the denotation $\epsilon_{n m}$ stands for the skew-symmetric Levi-Civita symbol. This expression (2.9) may be obtained as an Euler-Poisson expression for the Lagrange function

$$
L=\frac{e_{m n} u^{m} \dot{u}^{n}}{\|\boldsymbol{u}\|^{3}}-m\|\boldsymbol{u}\|
$$

The first addend in (2.10) is sometimes called the signed Frenet curvature [12] in $\mathbb{E}^{2}$. This, along with the observation that in two dimensional (pseudo)-Riemannian space the Frenet curvature

$$
k=\frac{\left\|\boldsymbol{u} \wedge \boldsymbol{u}^{\prime}\right\|}{\|\boldsymbol{u}\|^{3}}= \pm \sqrt{|g|} \frac{\epsilon_{k n} u^{k} u^{\prime n}}{\|\boldsymbol{u}\|^{3}}
$$

depends linearly on the covariant derivative $\boldsymbol{u}^{\prime}$ and thus produces at most the third order EulerPoisson equation, suggests the next assertion, based on Proposition 1:

Proposition $3([13,3])$. The variational functional with the Lagrange function

$$
L^{\mathcal{R}}=\sqrt{|g|} \frac{\epsilon_{k n} u^{k} u^{\prime n}}{\|\boldsymbol{u}\|^{3}}-m\|\boldsymbol{u}\|
$$

produces geodesic circles in two dimensional Riemannian space.

Note 2. The Lagrange function (2.10) looks like a relativistic analogue of the one recently treated in [14]. 


\section{The variational equation for geodesic circles}

\subsection{The generalized covariant momenta}

It is known that the Euler-Poisson expression (in this paper, - of the third order) (2.5) constitutes a covariant geometric object. But the conventional momentum $p_{n}$ from (2.4) does not. Therefore we introduce covariant momenta $\boldsymbol{\pi}$ and $\boldsymbol{\pi}^{(1)}$ that, in the case of the Lagrange function we shall deal with, represent some relative vectors in the following manner. First, we assume that the Lagrange function depends on the variables $\dot{u}^{n}$ exclusively through the covariant derivative $\boldsymbol{u}^{\prime}$. Moreover, in the case handled in this article, the partial derivatives $\frac{\partial L}{\partial \dot{u}^{n}}$ and $\frac{\partial L}{\partial u^{\prime n}}$ coincide. Let us introduce the "truncated" partial derivatives

$$
\frac{\overline{\partial L}}{\partial u^{n}}=\frac{\partial L}{\partial u^{n}}-\frac{\partial u^{\prime q}}{\partial u^{n}} \frac{\partial L}{\partial u^{\prime q}},
$$

and, for future use,

$$
\frac{\overline{\partial L}}{\partial x^{n}}=\frac{\partial L}{\partial x^{n}}-\frac{\partial u^{\prime q}}{\partial x^{n}} \frac{\partial L}{\partial u^{\prime q}} .
$$

Then the quantities

$$
\begin{aligned}
\boldsymbol{\pi}^{(1)} & =\frac{\partial L}{\partial \boldsymbol{u}^{\prime}} \quad \text { and } \\
\boldsymbol{\pi} & =\frac{\partial L}{\partial \boldsymbol{u}}-\boldsymbol{\pi}^{(1) \prime} \quad(\text { see }(3.1)) \\
& =\frac{\partial L}{\partial u^{n}}-2 \Gamma^{q}{ }_{m n} u^{m} \pi^{(1)}{ }_{q}-\pi^{(1) \prime} \quad \text { (by virtue of (A.5)) }
\end{aligned}
$$

may each be applied the operation of the covariant differentiation according to the formulae (A.5). For example, the covariant momentum $\boldsymbol{\pi}^{(1)}$ produces its covariant derivative

$$
\pi^{(1) \prime}{ }_{n}=\dot{\pi}^{(1)}{ }_{n}-\Gamma^{m}{ }_{l n} \pi^{(1)}{ }_{m} u^{l} .
$$

In the above terms the quantity $p_{n}$ of (2.4) is expressed as follows

$$
\begin{aligned}
p_{n}= & \left.\pi_{n}+2 \Gamma_{m n}^{q} u^{m} \pi^{(1)}+\pi^{(1) \prime} \quad \quad \text { (on base of }(3.5)\right) \\
& -\dot{\pi}^{(1)}{ }_{n} \quad(\text { on base of }(3.3)) \\
= & \pi_{n}+\Gamma^{q}{ }_{m n} u^{m} \pi_{q}^{(1)} \quad(\text { on base of }(3.6)) .
\end{aligned}
$$

Differentiating (3.7) and applying (A.5) to express the ordinary derivatives of $\pi_{n}$ and $u^{n}$ in terms of $\boldsymbol{\pi}^{\prime}$ and $\boldsymbol{u}^{\prime}$ along with (3.6), gives

$$
\begin{aligned}
\dot{p}_{n}= & \left(\pi_{n}^{\prime}+\Gamma_{m n}^{l} \pi_{l} u^{m}\right)+\frac{\partial \Gamma^{l}{ }_{m n}}{\partial x^{k}} u^{k} u^{m} \pi^{(1)}{ }_{l} \\
& +\left(\Gamma^{l}{ }_{m n} u^{\prime m}-\Gamma^{l}{ }_{m n} \Gamma^{m}{ }_{q k} u^{q} u^{k}\right) \pi^{(1)} l+\Gamma_{m n}^{l} u^{m}\left(\pi^{(1) \prime}{ }_{l}+\Gamma^{q}{ }_{k l} \pi^{(1)}{ }_{q} u^{k}\right) \\
= & \pi_{n}^{\prime}+\left(\pi^{(1) \prime}{ }_{l}+\pi_{l}\right) \Gamma_{m n}^{l}{ }_{m n} u^{m}+\pi^{(1)}{ }_{l} \Gamma_{m n} u^{\prime m} \\
& +\pi^{(1)}{ }_{q} u^{m} u^{k}\left(\Gamma^{l}{ }_{m n} \Gamma^{q}{ }_{l k}+\frac{\partial \Gamma^{q}{ }_{m n}}{\partial x^{k}}-\Gamma^{q}{ }_{l n} \Gamma^{l}{ }_{m k}\right) .
\end{aligned}
$$

Now the Euler-Poisson expression (2.5) may be handled with the help of our "truncated" $x$-derivative (3.2) in the following way

$$
\mathcal{E}_{n}=\frac{\overline{\partial L}}{\partial x^{n}}-\pi_{n}^{\prime}-\left(\pi^{(1) \prime}{ }_{l}+\pi_{l}\right) \Gamma^{l}{ }_{m n} u^{m}-\pi^{(1)}{ }_{l} \Gamma^{l}{ }_{m n} u^{\prime m}-\pi^{(1)}{ }_{l} u^{m} u^{k} R_{n k m}{ }^{l},
$$

making use of the definition of the curvature tensor (A.9). 


\subsection{The Euler-Poisson expression for the signed Frenet curvature}

Let us carry out the previous considerations taking for the Lagrange function $L_{\mathrm{II}}$ the signed Frenet curvature

$$
L_{\mathrm{II}}=\sqrt{|g|} \frac{\epsilon_{m n} u^{m} u^{\prime n}}{\|\boldsymbol{u}\|^{3}} .
$$

Making use of (A.7) and (A.6) one gets immediately

$$
\frac{\overline{\partial L_{\mathrm{II}}}}{\partial x^{n}}=\sqrt{|g|} \frac{\Gamma^{q}{ }_{q n} \epsilon_{m k} u^{m} u^{\prime k}}{\|\boldsymbol{u}\|^{3}}-3 \sqrt{|g|} \frac{\Gamma_{n m}^{l} u_{l} u^{m}}{\|\boldsymbol{u}\|^{5}} .
$$

The expression for $\boldsymbol{\pi}^{(1)}$ is obtained from (3.3) in still easier way

$$
\pi_{n}^{(1)}=\sqrt{|g|} \frac{\epsilon_{k n} u^{k}}{\|\boldsymbol{u}\|^{3}} .
$$

The covariant derivative of $\boldsymbol{\pi}^{(1)}$ is presented by the decomposition formula (3.6). While calculating $\dot{\pi}^{(1)}{ }_{n}$ in (3.6) we profit both from (A.7) and from (A.8). Then we replace $\pi^{(1)}{ }_{n}$ in (3.6) with (3.11) to obtain

$$
\begin{aligned}
\pi_{n}^{(1) \prime}= & \sqrt{|g|} \frac{\Gamma^{q}{ }_{q l} u^{l} \epsilon_{k n} u^{k}}{\|\boldsymbol{u}\|^{3}}+\sqrt{|g|} \frac{\epsilon_{k n}}{\|\boldsymbol{u}\|^{3}}\left(u^{\prime k}-\Gamma_{m l}^{k} u^{l} u^{m}\right) \\
& -3 \sqrt{|g|} \frac{\left(\boldsymbol{u} \cdot \boldsymbol{u}^{\prime}\right) \epsilon_{k n} u^{k}}{\|\boldsymbol{u}\|^{5}}-\sqrt{|g|} \frac{\Gamma_{n m}^{l} \epsilon_{k l} u^{k} u^{m}}{\|\boldsymbol{u}\|^{3}} .
\end{aligned}
$$

Finally, the simplification formula (A.4) may be used to produce

$$
\pi_{n}^{(1) \prime}=\sqrt{|g|} \frac{\epsilon_{k n} u^{\prime k}}{\|\boldsymbol{u}\|^{3}}-3 \sqrt{|g|} \frac{\left(\boldsymbol{u} \cdot \boldsymbol{u}^{\prime}\right) \epsilon_{k n} u^{k}}{\|\boldsymbol{u}\|^{5}} .
$$

The calculation of the sum $\boldsymbol{\pi}+\boldsymbol{\pi}^{(1) \boldsymbol{\prime}}$ on grounds of (3.4) is straightforward

$$
\pi_{n}+\pi_{n}^{(1) \prime}=-\sqrt{|g|} \frac{\epsilon_{k n} u^{\prime k}}{\|\boldsymbol{u}\|^{3}}-3 \sqrt{|g|} \frac{u_{n} \epsilon_{l k} u^{l} u^{\prime k}}{\|\boldsymbol{u}\|^{5}} .
$$

Or, alternatively, utilizing (A.3), one obtains

$$
\pi_{n}+\pi_{n}^{(1) \prime}=2 \sqrt{|g|} \frac{\epsilon_{k n} u^{\prime k}}{\|\boldsymbol{u}\|^{3}}-3 \sqrt{|g|} \frac{\left(\boldsymbol{u} \cdot \boldsymbol{u}^{\prime}\right) \epsilon_{k n} u^{k}}{\|\boldsymbol{u}\|^{5}} .
$$

To calculate $\boldsymbol{\pi}$, we extract (3.13) from (3.14)

$$
\pi_{n}=\sqrt{|g|} \frac{\epsilon_{k n} u^{\prime k}}{\|\boldsymbol{u}\|^{3}} .
$$

Now, the Euler-Poisson expression (3.8) for the Lagrange function (3.9) by virtue of (3.10), (3.11), and (3.14) reduces to

$$
\mathcal{E}_{n}\left(L_{\mathrm{II}}\right)=-\pi_{n}^{\prime}-\pi^{(1)}{ }_{q} R_{n k m}{ }^{q} u^{m} u^{k},
$$

where the covariant derivative of the momentum $\boldsymbol{\pi}$ may be calculated starting with the definition (A.5) applied to (3.15), wherein $\left(u^{\prime k}\right)^{\bullet}$ should be calculated again along the lines (A.5)

$$
\left(u^{\prime k}\right)^{\bullet}=u^{\prime \prime k}-\Gamma_{l m}^{k} u^{\prime m} u^{l} .
$$


Using then (A.7) and (A.8) along with the simplification formula (A.4) once more, we calculate

$$
\pi_{n}^{\prime}=-3 \frac{\sqrt{|g|}\left(\boldsymbol{u} \cdot \boldsymbol{u}^{\prime}\right)}{\|\boldsymbol{u}\|^{5}} \epsilon_{k n} u^{\prime k}+\frac{\sqrt{|g|}}{\|\boldsymbol{u}\|^{3}} \epsilon_{k n} u^{\prime \prime k} .
$$

Thus in the disclosed form the Euler-Poisson expression for the total Lagrange function (2.12) reads

$$
\begin{aligned}
\mathcal{E}_{n}^{\mathcal{R}}= & -\sqrt{|g|} \frac{\epsilon_{k n} u^{\prime \prime k}}{\|\boldsymbol{u}\|^{3}}+3 \sqrt{|g|} \frac{\left(\boldsymbol{u} \cdot \boldsymbol{u}^{\prime}\right) \epsilon_{k n} u^{\prime k}}{\|\boldsymbol{u}\|^{5}} \\
& +m \frac{(\boldsymbol{u} \cdot \boldsymbol{u}) u^{\prime}{ }_{n}-\left(\boldsymbol{u}^{\prime} \cdot \boldsymbol{u}\right) u_{n}}{\|\boldsymbol{u}\|^{3}}-\frac{\sqrt{|g|}}{\|\boldsymbol{u}\|^{3}} \epsilon_{m q} R_{n k l}{ }^{q} u^{m} u^{l} u^{k} .
\end{aligned}
$$

The Euler-Poisson equation, solved with respect to highest derivative, now is

$$
\sqrt{|g|}\left(\frac{u^{\prime \prime k}}{\|\boldsymbol{u}\|^{3}}-3 \frac{\left(\boldsymbol{u} \cdot \boldsymbol{u}^{\prime}\right) u^{\prime k}}{\|\boldsymbol{u}\|^{5}}\right)-m \frac{(\boldsymbol{u} \cdot \boldsymbol{u}) e^{n k} u_{n}^{\prime}-\left(\boldsymbol{u}^{\prime} \cdot \boldsymbol{u}\right) e^{n k} u_{n}}{\|\boldsymbol{u}\|^{3}}+e^{n k} \mathcal{R}_{n}=0
$$

where we used the notion of the contravariant skew-symmetric Levi-Civita symbol $e^{m n}=$ $\operatorname{det}\left[g_{p q}\right] g^{m k} g^{n l} \epsilon_{k l}$, and introduced the shortcut

$$
\mathcal{R}_{n}=\frac{\sqrt{|g|}}{\|\boldsymbol{u}\|^{3}} \epsilon_{m q} R_{n k l} u^{m} u^{l} u^{k}
$$

to denote the force on the particle, evoked by the curvature of the (pseudo)-Riemannian structure. Equation (3.17) obviously generalizes that of (2.9).

Note 3. If, on the other hand, we had introduced a spin tensor $S_{m q}=\pi^{(1)}{ }_{m} u_{q}-\pi^{(1)}{ }_{q} u_{m}$, the second term on the right of formula (3.16) would have got an interpretation as the force, evoked by the existence of quasi-classical spin, and would coincide with that present in Dixon's equation [15].

Proposition 4. The differential equation (3.18) describes all geodesic circles.

Proof. We have to show that, for each solution of (1.1) it is always possible to perform such transformation of the independent variable $s$ to $\xi$ that after that change of variables the equation (3.18) will hold.

First, let us rewrite the differential equation of geodesic circles (1.1) in arbitrary parametrization

$$
\frac{\boldsymbol{u}^{\prime \prime}}{\|\boldsymbol{u}\|^{3}}=\frac{\boldsymbol{u} \cdot \boldsymbol{u}^{\prime \prime}}{\|\boldsymbol{u}\|^{5}} \boldsymbol{u}+3 \frac{\left(\boldsymbol{u} \cdot \boldsymbol{u}^{\prime}\right)}{\|\boldsymbol{u}\|^{5}} \boldsymbol{u}^{\prime}-3 \frac{\left(\boldsymbol{u} \cdot \boldsymbol{u}^{\prime}\right)^{2}}{\|\boldsymbol{u}\|^{7}} \boldsymbol{u} .
$$

We add to it one more equation, which will play the role of such a one, that fixes the parametrization along the curve

$$
\sqrt{|g|}\left(\frac{\boldsymbol{u} \cdot \boldsymbol{u}^{\prime \prime}}{\|\boldsymbol{u}\|^{3}}-3 \frac{\left(\boldsymbol{u} \cdot \boldsymbol{u}^{\prime}\right)^{2}}{\|\boldsymbol{u}\|^{5}}\right)=e^{n k}\left(\frac{m}{\|\boldsymbol{u}\|} u_{n}^{\prime} u_{k}-u_{k} \mathcal{R}_{n}\right) .
$$

This equation is consistent with the equation (3.18) because it presents nothing more than a mere consequence of (3.18), obtained by means of the contraction with $u_{k}$. Further, let us substitute $\frac{u^{\prime \prime k}}{\|\boldsymbol{u}\|^{3}}$ in (3.18) from the equation (3.20)

$$
-\sqrt{|g|}\left(\frac{\boldsymbol{u} \cdot \boldsymbol{u}^{\prime \prime}}{\|\boldsymbol{u}\|^{5}}-3 \frac{\left(\boldsymbol{u} \cdot \boldsymbol{u}^{\prime}\right)^{2}}{\|\boldsymbol{u}\|^{7}}\right) u^{k}=e^{k n}\left(m \frac{(\boldsymbol{u} \cdot \boldsymbol{u}) u_{n}^{\prime}+\left(\boldsymbol{u}^{\prime} \cdot \boldsymbol{u}\right) u_{n}}{\|\boldsymbol{u}\|^{3}}-\mathcal{R}_{n}\right) .
$$


It remains to insert the left hand side of (3.21) into the left hand side of (3.22) and to notice that in two-dimensional case what comes out is the identity

$$
\frac{1}{\boldsymbol{u} \cdot \boldsymbol{u}}\left(\frac{m}{\|\boldsymbol{u}\|} e^{n l} u_{n}^{\prime} u_{l}-e^{n l} u_{l} \mathcal{R}_{n}\right) u^{k} \equiv e^{k n}\left(\frac{m}{\|\boldsymbol{u}\|} u_{n}^{\prime}-m \frac{\boldsymbol{u}^{\prime} \cdot \boldsymbol{u}}{\|\boldsymbol{u}\|^{3}} u_{n}-\mathcal{R}_{n}\right) .
$$

To see that (3.23) is satisfied identically, it suffices to apply the simplification formula (A.3) separately to the terms involving $\mathcal{R}$ and then to what remains, and to notice that $u^{n} \mathcal{R}_{n}=0$ due to the previously introduced notation (3.19).

\section{A Appendix}

\section{A.1 Parameter independence and fundamental fields}

The $T^{r} M$ : loose comments. In the variational calculus various objects depend not only on the variables of the configuration space, but also on the velocities and on the accelerations of the first as well as of higher orders. The space of this extended number of variables may be introduced in different ways, as it has in fact been by different authors. In present paper we choose to use the definition, belonging to Ehresmann [17], of the higher order velocity space $T^{r} M=J^{r}{ }_{0}(\mathbb{R}, M)_{0}$ as the set of jets of mappings from a neighborhood of the origin in $\mathbb{R}$ to the configuration space $M=x^{n}$, which all start at the origin $0 \in \mathbb{R}$. This space $T^{r} M$ is naturally endowed by several geometric structures. The Reader may consult the monographs [16] and [9] on the subject. For the purposes of this paper it suffices to think about $T^{r} M$ as a manifold of the variables $x^{n}, u^{n}, \ldots, u_{r-1}^{n}$, constructed of the successive derivatives of the configuration space variables $x^{n}$ by the independent evolutionary one.

The fundamental fields. The group of invertible jets $\tilde{J}^{2}{ }_{0}(\mathbb{R}, \mathbb{R})_{0}$ with the source as well as the target at the origin $0 \in \mathbb{R}$ presents an appropriate geometrical concept when speaking about local transformations of the parameter along the curve in a manifold. Let again the variables $u^{n}=\dot{x}^{n}, \dot{u}^{n}=\ddot{x}^{n}$ denote the first and the second derivatives of coordinates along the curve $x^{n}(\xi)$, so that the jet $j_{0}^{(2)} x$ at zero is presented by the array of second-order polynomials $u^{n} \xi+\dot{u}^{n} \xi^{2}$. Another jet, $j^{(2)} \sigma \in \tilde{J}_{0}^{2}(\mathbb{R}, \mathbb{R})_{0}$, which is presented by the polynomial $\alpha \xi+\frac{1}{2} \beta \xi^{2}$, acts on the right upon the previous one by the composition

$$
\begin{aligned}
j_{0}^{(2)} x \cdot j^{(2)} \sigma & =j^{(2)}(x \circ \sigma)=u^{n} \cdot\left(\alpha \xi+\frac{1}{2} \beta \xi^{2}\right)+\frac{1}{2} \dot{u}^{n} \cdot\left(\alpha \xi+\frac{1}{2} \beta \xi^{2}\right)^{2} \\
& =u^{n} \alpha \xi+\frac{1}{2}\left(u^{n} \beta+\dot{u}^{n} \alpha^{2}\right) \xi^{2} \quad \bmod o\left(\xi^{2}\right) .
\end{aligned}
$$

Consider an $\varepsilon$-shift $\sigma_{\varepsilon}(\xi)$ of the transformation $\sigma$ of the independent variable $\xi$ in the local curve expression $x^{n}(\xi)$. This shift is presented in $\tilde{J}_{0}^{2}(\mathbb{R}, \mathbb{R})_{0}$ by some $\alpha_{\varepsilon}=\frac{\partial \sigma_{\varepsilon}}{\partial \xi}(0)$ and $\beta_{\varepsilon}=\frac{\partial^{2} \sigma_{\varepsilon}}{\partial \xi^{2}}(0)$. It evokes a corresponding flow $\left\{u_{\varepsilon}^{n}, \dot{u}_{\varepsilon}^{n}\right\} \in T^{2} M$ induced by the action (A.1)

$$
u_{\varepsilon}^{n}=\alpha_{\varepsilon} u^{n}, \quad \dot{u}_{\varepsilon}^{n}=\beta_{\varepsilon} u^{n}+\alpha_{\varepsilon}^{2} \dot{u}^{n} .
$$

The generator of this flow is

$$
\left.u^{n} \frac{d \alpha_{\varepsilon}}{d \varepsilon}\right|_{\varepsilon=0} \frac{\partial}{\partial u^{n}}+\left.\left(\frac{d \beta_{\varepsilon}}{d \varepsilon} u^{n}+2 \alpha_{\varepsilon} \frac{d \alpha_{\varepsilon}}{d \varepsilon} \dot{u}^{n}\right)\right|_{\varepsilon=0} \frac{\partial}{\partial \dot{u}^{n}} .
$$

Now it suffices to limit ourselves to the shift $\sigma_{\varepsilon}(\xi)$ of the form $\sigma_{\varepsilon}(\xi)=\xi+\varepsilon \tau(\xi)$. Then the generator (A.2) reads

$$
u^{n} \frac{d \tau}{d \xi}(0) \frac{\partial}{\partial u^{n}}+\left(u^{n} \frac{d^{2} \tau}{d \xi^{2}}(0)+2 \dot{u}^{n} \frac{d \tau}{d \xi}(0)\right) \frac{\partial}{\partial \dot{u}^{n}} .
$$

Taking $\tau(\xi)=\xi$ we obtain the first fundamental field $\zeta_{1}$ in (2.1). Choosing $\tau(\xi)=\frac{1}{2} \xi^{2}$ we obtain the second fundamental field $\zeta_{2}$ in (2.1). 


\section{A.2 The inverse variational problem: loose comments}

The system of equations (2.7) along with the specific guise (2.6) of the Euler-Poisson equation arises as the general solution of the so-called inverse variational problem in the calculus of variations. This problem has been attacked by numerous authors from varying points of view based on different approaches. Roughly speaking, the problem consists in finding out the criterion that for an a priori given differential equation there locally exists a Lagrange function, from which this equation follows by the variational procedure, applied to the corresponding action functional. As far as the act of the variation of the functional may be expressed in the form of some operator $\delta$ action on the Lagrange function $L$ in such a way that $\mathcal{E}=\delta L$ be a well-defined geometric object, represented in local coordinates by means of well-known system of the Euler-Poisson expressions $\mathcal{E}_{n}$, it is tempting to give such a definition of $\delta$, wherewith the cohomology complex property $\delta^{2}$ should hold. The conditions (2.7) express the fact that the differential form $\mathcal{E}_{n} d x^{n}$ is closed with respect to $\delta$. It is not our intention in this paper to discuss further the ways of defining the operator $\delta$. Along with the sources $[10,11]$ we wish to show the interested reader to the book by Olga Krupková [18] with the plentitude of references therein. However, our explanation here was based on the approach of Tulczyjew [19].

\section{A.3 Simplifications of exterior products}

In two dimensions some vector and tensor skew-symmetric expressions simplify drastically. Let $a^{n}, b^{n}$ and $c^{n}$ denote arbitrary vectors and let $\Gamma_{m n}^{l}$ denote for a moment an arbitrary three-index quantity. The following two simplifications keep true if the underlying (pseudo)Riemannian manifold is two-dimensional

$$
\begin{aligned}
& g_{m n} a^{m} a^{n} \epsilon_{l k} b^{l}-g_{m n} a^{m} b^{n} \epsilon_{l k} a^{l}+a_{k} \epsilon_{m n} a^{m} b^{n}=0, \\
& \epsilon_{m n} a^{m} b^{n} \Gamma_{l k}^{l} c^{k}-\epsilon_{m n} b^{n} \Gamma^{m}{ }_{l k} a^{l} c^{k}+\epsilon_{m n} a^{n} \Gamma^{m} l k b^{l} c^{k}=0 .
\end{aligned}
$$

The proof consists in the ingenuous calculation.

\section{A.4 Formulae from (pseudo)-Riemannian geometry}

Here we list some well-known relations, involving Christoffel symbols, curvature tensor, and the covariant derivative. The latter will be denoted by the prime superscript, while to denote the ordinary derivative the dot will be used, e.g. $u^{n}=\dot{x}^{n}, \dot{u}^{n}=\ddot{x}^{n}$, etc.

$$
\begin{aligned}
& a^{\prime n}=\dot{a}^{n}+\Gamma_{l m}^{n} a^{m} u^{l}, \quad a_{n}^{\prime}=\dot{a}_{n}-\Gamma_{l n}^{m} a_{m} u^{l}, \\
& \frac{\partial g_{m n}}{\partial x^{k}}=g_{m l} \Gamma_{k n}^{l}+g_{n l} \Gamma_{k m}^{l}, \\
& \frac{\partial}{\partial x^{n}} \sqrt{|g|}=\sqrt{|g|} \Gamma^{l}{ }_{l n}, \quad \text { where } \quad g=\operatorname{det}\left[g_{n m}\right], \\
& \left(\frac{1}{\|\boldsymbol{u}\|^{3}}\right)^{\prime}=-3 \frac{\boldsymbol{u} \cdot \boldsymbol{u}^{\prime}}{\|\boldsymbol{u}\|^{5}}, \\
& R_{k m n}{ }^{l}=\frac{\partial \Gamma_{k n}^{l}}{\partial x^{m}}-\frac{\partial \Gamma_{m n}^{l}}{\partial x^{k}}+\Gamma_{m q}^{l} \Gamma_{k n}^{q}-\Gamma_{k q}^{l} \Gamma_{m n}^{q} .
\end{aligned}
$$

\section{A.5 Characterization of geodesic circles}

It is not difficult to check that the condition $\frac{d k}{d \xi}=0$ in two dimensions is equal to the equation (1.1) on the shell $g_{m n} u^{m} u^{n}=1$. More precisely, at the constraint manifold

$$
\boldsymbol{u} \cdot \boldsymbol{u}=1
$$




$$
\begin{aligned}
& \boldsymbol{u} \cdot \boldsymbol{u}^{\prime}=0, \\
& \boldsymbol{u}^{\prime} \cdot \boldsymbol{u}^{\prime}+\boldsymbol{u} \cdot \boldsymbol{u}^{\prime \prime}=0,
\end{aligned}
$$

the differential equation

$$
k^{2 \prime} \equiv \pm \boldsymbol{u}^{\prime} \cdot \boldsymbol{u}^{\prime \prime}=0
$$

is equivalent to the differential equation of the geodesic circles

$$
\boldsymbol{u}^{\prime \prime}+\left(\boldsymbol{u}^{\prime} \cdot \boldsymbol{u}^{\prime}\right) \boldsymbol{u}=\mathbf{0}
$$

In fact, from (A.13) and (A.12) one solves for $\boldsymbol{u}^{\prime \prime}$

$$
u_{l}^{\prime \prime}=\frac{\epsilon_{l m} u^{\prime m}}{\epsilon_{m n} u^{\prime m} u^{n}} \boldsymbol{u}^{\prime} \cdot \boldsymbol{u}^{\prime}
$$

and then implementing (A.3) with the help of (A.10) and (A.11) obtains (A.14). Conversely, the (A.13) in nothing but the (A.14) contracted with $\boldsymbol{u}^{\prime}$ at the constraint manifold (A.11).

\section{Acknowledgements}

This work was supported by the Grant GAČR 201/06/0922 of Czech Science Foundation.

\section{References}

[1] Hill E.L., On the kinematics of uniformly accelerated motions and classical magnetic theory, Phys. Rev. 72 (1947), 143-149.

[2] Yano K., Concircular geometry I. Concircular transformations, Proc. Imp. Acad. Jap. 16 (1940), 195-200.

[3] Matsyuk R.Ya., Variational principle for uniformly accelerated motion, Mat. Metody Fiz.-Mekh. Polya 16 (1982), 84-88 (in Russian).

[4] Arodź H., Sitarz A., Wȩgrzyn P., On relativistic point particles with curvature-dependent actions, Acta Phys. Polon. B 20 (1989), 921-939.

[5] Matsyuk R.Ya., Poincaré-invariant equations of motion in Lagrangian mechanics with higher derivatives, PhD Thesis, Institute for Applied Problems in Mechanics and Mathematics, L'viv, 1984 (in Russian).

[6] Logan J.D., Invariant variational principles, Academic Press, New York, 1977.

[7] Kawaguchi M., An introduction to the theory of higher order spaces. II. Higher order spaces in multiple parameters, RAAG Memoirs 4 (1968), 578-592.

[8] Matsyuk R.Ya., Autoparallel variational description of the free relativistic top third order dynamics, in Proceedings of Eight International Conference "Differential Geometry and Its Applications" (August 27-31, 2001, Opava, Czech Republic), Editors O. Kowalski et al., Silesian Univ., Opava, 2002, 447-452.

[9] de Léon M., Rodrigues P. R., Generalized classical mechanics and field theory, Elsevier, Amsterdam, 1985.

[10] Matsyuk R.Ya., On the existence of a Lagrangian for a system of ordinary differential equations, Mat. Metody Fiz.-Mekh. Polya 13 (1981), 34-38, 113 (in Russian).

[11] Matsyuk R.Ya., Lagrangian analysis of invariant third-order equations of motion in relativistic classical particle mechanics, Dokl. Akad. Nauk SSSR 282 (1985), 841-844 (English transl.: Soviet Phys. Dokl. 30 (1985), 458-460).

[12] Arreaga G., Capovilla R., Guven J., Frenet-Serret dynamics, Classical Quantum Gravity 18 (2001), 50655083, hep-th/0105040.

[13] Matsyuk R.Ya., The variational principle for geodesic circles, in Boundary Value Problems of Mathematical Physics, Naukova Dumka, Kiev, 1981, 79-81 (in Russian).

[14] Acatrinei C.S., A path integral leading to higher order Lagrangians, J. Phys. A: Math. Gen. 40 (2007), F929-F933, arXiv:0708.4351. 
[15] Dixon W.G., Dynamics of extended bodies in general relativity I. Momentum and angular momentum, Proc. Roy. Soc. London. Ser. A. 314 (1970), 499-527.

[16] Yano K., Ishihara Sh., Tangent and cotangent bundles, Marcel Dekker, New York, 1973.

[17] Ehresmann Ch., Les prolongements dúne variété différentiable I. Calcul des jets, prolongement principal, C. R. Math. Acad. Sci. Paris 233 (1951), 598-600.

[18] Kruprová O., The geometry of ordinary variational equations, Lect. Notes in Math., Vol. 1678, SpringerVerlag, Berlin, 1997.

[19] Tulczyjew W.M., Sur la différentielle de Lagrange, C. R. Math. Acad. Sci. Paris 280 (1975), $1295-1298$. 